\title{
Real-time, multidimensional in vivo imaging used to investigate blood flow in mouse pancreatic islets
}

\author{
Lara R. Nyman, ${ }^{1}$ K. Sam Wells, ${ }^{2}$ W. Steve Head, ${ }^{2}$ Michael McCaughey, ${ }^{2}$ \\ Eric Ford, ${ }^{1}$ Marcela Brissova, ${ }^{1}$ David W. Piston, ${ }^{2}$ and Alvin C. Powers ${ }^{1,2,3}$ \\ 1Department of Medicine and 2Department of Molecular Physiology and Biophysics, Vanderbilt University, Nashville, Tennessee, USA. \\ 3VA Tennessee Valley Healthcare System, Nashville, Tennessee, USA.
}

\begin{abstract}
The pancreatic islets of Langerhans are highly vascularized micro-organs that play a key role in the regulation of blood glucose homeostasis. The specific arrangement of endocrine cell types in islets suggests a coupling between morphology and function within the islet. Here, we established a line-scanning confocal microscopy approach to examine the relationship between blood flow and islet cell type arrangement by real-time in vivo imaging of intra-islet blood flow in mice. These data were used to reconstruct the in vivo 3D architecture of the islet and time-resolved blood flow patterns throughout the islet vascular bed. The results revealed 2 predominant blood flow patterns in mouse islets: inner-to-outer, in which blood perfuses the core of $\beta$ cells before the islet perimeter of non- $\beta$ cells, and top-to-bottom, in which blood perfuses the islet from one side to the other regardless of cell type. Our approach included both millisecond temporal resolution and submicron spatial resolution, allowing for real-time imaging of islet blood flow within the living mouse, which has not to our knowledge been attainable by other methods.
\end{abstract}

\section{Introduction}

Irregularities in pancreatic islet blood flow have been postulated to play a major role in islet pathophysiology as well as in the failure of islet transplants (1-5). Pancreatic islets are highly vascularized micro-organs with a central core of insulin-producing $\beta$ cells surrounded by a perimeter of non- $\beta$ islet cells, which secrete glucagon, somatostatin, pancreatic polypeptide, and ghrelin $(6,7)$. The vascular network within the islet consists of vessels that are wider, more numerous, and more tortuous than those of the surrounding exocrine tissue $(5,8)$. It has been suggested that the distinct arrangement of the different cell types and the patterns of blood flow in the islet are functionally coupled. This relationship has important physiologic implications, since both in vitro and in vivo studies indicate that products secreted by one islet cell type can influence hormone secretion by other types of islet cells (9-12).

Previous work has proposed 3 distinct, yet mutually exclusive, models for islet blood flow. In one model, blood flows first to the non- $\beta$ islet cells on the perimeter and then to the $\beta$ cells in the islet core (13-16). This model is based on results of structural studies using scanning electron microscopy of corrosion vascular casts, which suggested that afferent vessels entering the non- $\beta$ cell perimeter branch into smaller vessels and perfuse the non- $\beta$ cells first (17). Consequently, products of islet perimeter cells would be secreted upstream of $\beta$ cells. In a contrasting model, afferent arterial flow bypasses the non- $\beta$ cell perimeter islet cells and branches into capillaries only after reaching the $\beta$ cell core; thus, blood would perfuse $\beta$ cells before non- $\beta$ cells. This model is also based on evidence from scanning electron microscopy of corrosion vascular casts (13) as well as India ink

Nonstandard abbreviations used: $\mathrm{fps}$, frame(s) per second; MIP, mouse insulin I promoter; ROI, region of interest.

Conflict of interest: The authors have declared that no conflict of interest exists. Citation for this article: J. Clin. Invest. 118:3790-3797 (2008). doi:10.1172/JCI36209. infusion studies and serial reconstructions of stained sections $(8$, 18). Additionally, retrograde and anterograde perfusions of the pancreas with neutralizing antibodies have provided a substantial body of physiological evidence for this $\beta$ cell-first perfusion theory $(11,12,19)$. Based on scanning electron microscopy as well as in vivo microscopy studies, a third model proposes that blood flow enters through a feeding artery on one side of the islet and immediately branches into multiple smaller vessels that perfuse blood from one side of the islet to the other regardless of cell type $(20,21)$. Because each predicts a different order for blood flow in relation to islet cell type, these models have significant implications for our understanding of islet hormone secretion and glucose homeostasis.

Until now, submicron resolution in vivo imaging studies have been limited. However, in vivo imaging offers the ability to examine tissues while they are actually functioning as a whole organ rather than as separated, nonliving tissue. This is especially important for understanding mechanisms and therapies for disease: often, data obtained in vitro are quite different than those obtained in vivo (22). Although there have been multiple studies using imaging modalities such as PET and MRI, these techniques do not offer sufficient spatial and temporal resolution to reveal the precise dynamics of blood flow at the capillary level within the pancreatic islet. In a recent publication, optical microscopy was used to assess pancreatic islet transplants in the anterior chamber of the eye, a transparent tissue, but dynamic studies were not reported (23).

Here we describe the development and application of a dynamic optical imaging approach to examine the relationship between islet morphology and vascular networks in vivo. To locate islets for imaging, we used the mouse insulin I promoter-GFP (MIP-GFP) mouse, a transgenic line with fluorescent green $\beta$ cells (24). This animal model was used with submicron spatial resolution and millisecond temporal resolution provided by a fast line-scan- 
A

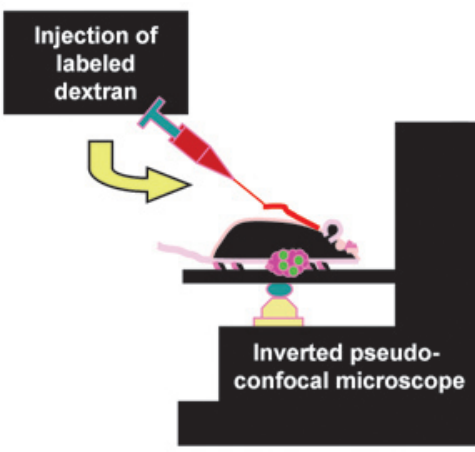

B

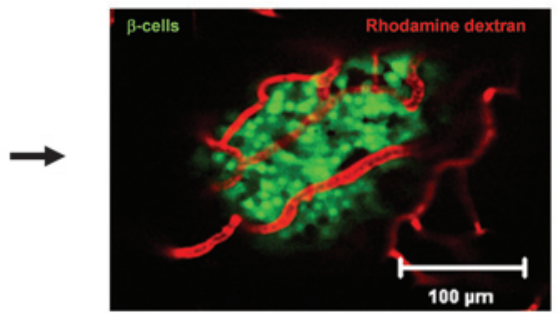

Figure 1

Imaging islet blood flow in vivo. (A) Schematic of in vivo imaging setup shows the positioning of an anesthetized MIP-GFP mouse with exteriorized pancreas over the lens of a high-speed, line-scanning confocal microscope. After epifluorescence visual identification of a GFP-labeled islet, rhodamine dextran was injected into the ocular plexus for visualization of islet blood in vivo. (B) Single-plane image of an islet with GFP-labeled $\beta$ cells (green); islet vasculature was visualized with rhodamine dextran (red) tracer injection. Note the dark areas within vessels, reflecting red blood cells that were not labeled with the rhodamine dextran. Also note that the surrounding exocrine tissue had fewer vessels than the islet. Scale bar: $100 \mu \mathrm{m}$.

ning confocal microscope, an approach that allowed us to image blood flow in vivo at extremely high speeds without sacrificing resolution. We developed this approach for quantitative fluorescence imaging, which we believe to be novel, and used it to address the controversy regarding islet blood flow perfusion order. Our successful application of this approach indicates that it will also be useful in investigating in vivo regulation of blood flow dynamics in islets as well as other organs.

\section{Results}

Imaging islet blood flow in vivo. As shown in Figure 1A, pancreatic islets and their microcirculation were imaged in vivo using high-speed, line-scanning confocal fluorescence microscopy (25, 26), a transgenic mouse line with EGFP-expressing $\beta$ cells (24), and intravenously injected rhodamine dextran (27). To achieve the spatial and temporal resolution needed to investigate islet microcirculation, the pancreas of the anesthetized mouse was exteriorized and oriented on the microscope stage so that the distance between the islet and the objective lens was minimized. High-resolution images of the islet and its functional vasculature within the intact pancreas confirmed the greater blood flow to pancreatic islets compared with surrounding pancreatic exocrine tissue (Figure 1B).

Assessment of pancreatic islet vascular structure in vivo. To facilitate the assessment of blood flow patterns within each islet, we imaged medium to large islets, ranging $160-500 \mu \mathrm{m}$ in diameter. A series of optical slices, with images focused 8-11 $\mu \mathrm{m}$ apart, were acquired within a partial volume of each islet, covering a focal depth of 25-50 $\mu \mathrm{m}$ (Figure 2). This made it possible to discern the location and size of individual vessels in relation to the $\beta$ cell mass throughout this subvolume of the islet. Through-focus projections (2-dimensional representations of a series of images taken at different focal planes) of the 3D data sets - commonly referred to as $z$ stacks - were used to visualize the overall islet structure, although all analyses were performed on individual image planes in order to discern the exact location of both large and small vessels in relation to the $\beta$ cells (Figure 3 ). While variations in microvascular structure were found, this heterogeneity did not correlate with islet size. Of 103 islets, 32 were associated with a larger vessel in the imaged portion of the islet. These larger vessels (>15 $\mu \mathrm{m}$ in diameter) were found to have multiple smaller vessels extending from them (Figure 3, arrows) and were located on the outer surfaces of the islet (Figure 2, A-C, arrows). In contrast, there were also islets that did not appear to be closely associated with any large vessel (Figure 3, bottom panels). When we analyzed individual image planes of this type, that is, an islet not associated with a large vessel, the islet vessel diameters were mostly homogeneous - dissimilar to the structures of graduated branching seen in islets containing or connected with a large vessel.

Assessment of afferent and efferent flow in the pancreatic islet. Because of the tortuous nature of the islet microvasculature and the high speeds of capillary flow, it has proven difficult to follow flow patterns into the islet. To address these issues, we acquired a time series of $z$ stacks. Optical slice spacing was set to achieve an imaging rate of 70-72 frames per second (fps), which we found to be the minimum frame speed needed to assess 3D blood flow patterns in most islets. Larger vessels with smaller branching capillaries, as described above, were found to be efferent rather than afferent vessels, as shown by both through-focus projection time series (Figure 4 and Supplemental Video 1; supplemental material available online with this article; doi:10.1172/JCI36209DS1) and single-plane time series (Supplemental Video 2). The time series data demonstrated that the bolus injection of rhodamine dextran reached these larger vessels after perfusion of the smaller vessels in the islet core (Figure 4, arrows). The real-time investigation of blood flow showed that most of the initial afferent flow into the islets was via smaller vessels (Supplemental Video 2). Of these 32 islets associated with larger vessels in the imaged portion, 11 were connected to the large vessel via numerous smaller branches. The large vessels in 9 of 11 exhibited efferent flow, 1 flow pattern was indiscernible, and 1 showed afferent flow. The 23 remaining islets were connected to a larger vessel by 1 or 2 smaller vessels, approximately the same diameter as islet capillaries, some of which exhibited afferent flow and others of which demonstrated efferent flow.

Assessment of islet microcirculatory patterns by fluorescence time course analysis. To determine the directional blood flow patterns in an islet, we measured the average fluorescence intensities over 
A

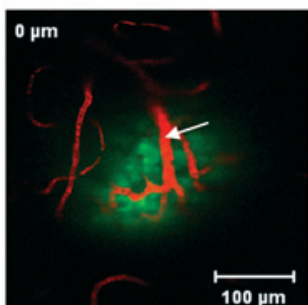

B

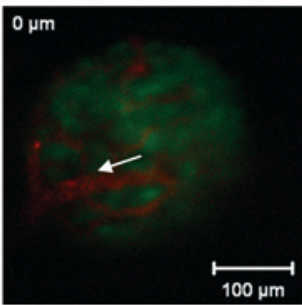

C

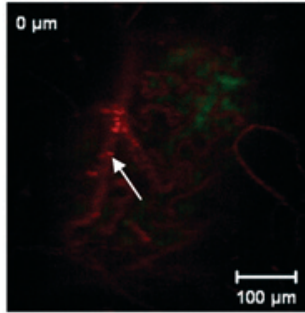

D

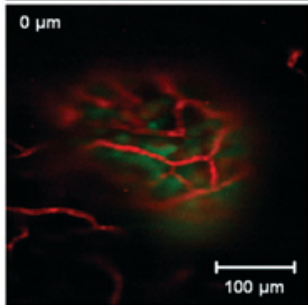

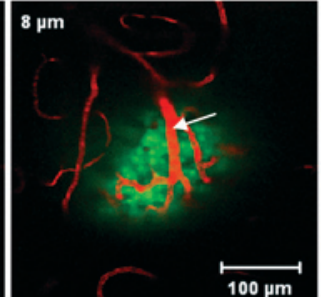
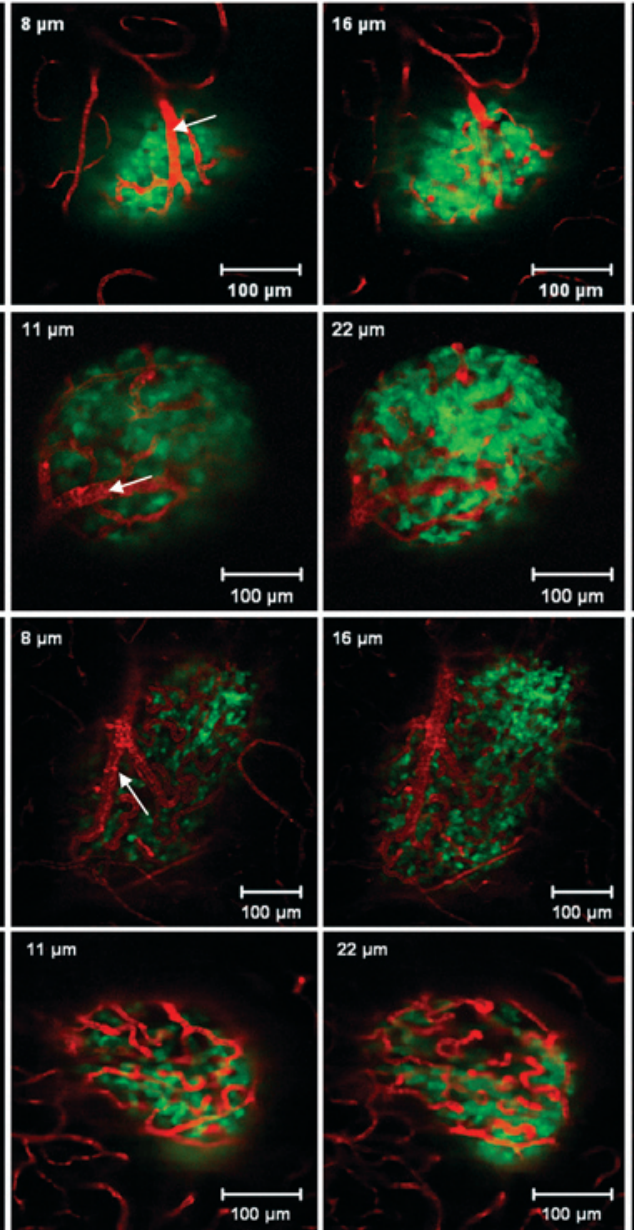
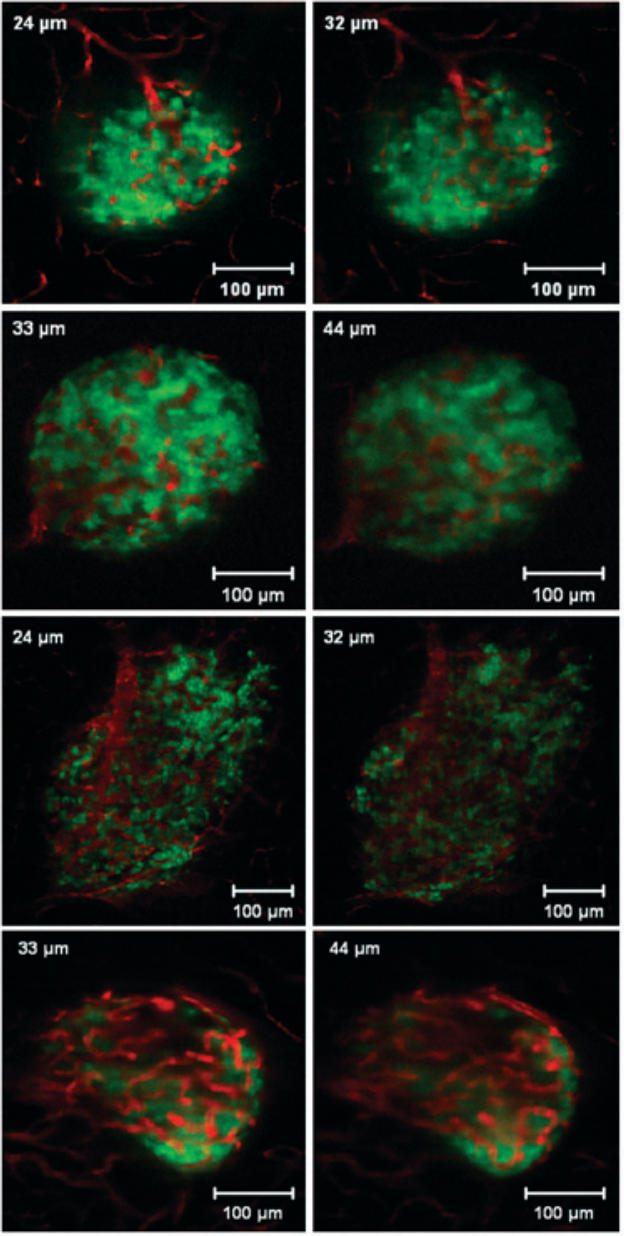

Figure 2

Variations in islet vascular structure in vivo. Shown are 5 optical slices spaced 8-11 $\mu \mathrm{m}$ apart along the $z$ direction for 4 different islets with rhodamine dextran-labeled plasma, demonstrating that vascular patterns differ in size and structure. The $z$ position relative to the first image plane (nearest to the coverglass) of the islet is noted in microns, while the $x y$ dimension is noted by $100-\mu \mathrm{m}$ scale bars. (A) Medium islet, containing a large vessel with numerous capillaries coalescing into it in the top image planes (arrows). (B and C) Larger islets of different shapes, which also had large, arm-like vessels in the top 2 planes (arrows). (D) Islet not closely associated with an arm-like vessel. Image slices throughout the $z$ plane showed vessel sizes to be mostly homogeneous in diameter, rather than having a graduated size pattern.

time in marked regions of interest (ROIs) within the vasculature throughout the 3D data set after bolus injection of rhodamine dextran. After defining inner and outer areas of the islet (Figure 5, A and C), intensity time course averages for inner vessels were measured and compared with those for outer vessel regions in multiple islets (Figure 5, B and D). To compare the time of entry of the rhodamine dextran into different parts of the islet vasculature, we used the initial rise time of fluorescence intensity for each region. The arrival time of a bolus at each of the selected ROIs was determined by finding the first time point in each time course after which the intensity of each following time point increased monotonically until the first local maximum was reached. These initial rise times were then used as indicators of the arrival of blood flow in the ROI.

There were 2 predominant patterns of pancreatic islet blood flow. In some islets, the initial rise time in fluorescence intensity for the vessels in the islet core was before that of vessels on the perimeter (Figure 5B, Supplemental Video 3, and Supplemental
Figure 1A), whereas other islets exhibited no difference between the average initial rise times of vessels in the core and the perimeter regions (Figure 5D, Supplemental Video 4, and Supplemental Figure $1 \mathrm{~B})$. This initial rise time was determined and then plotted for each core- or perimeter-marked ROI throughout the volume of each islet (Figure 6). The majority of islets (12 of $20 ; 60 \%$ ) demonstrated an "inner-to-outer" pattern, indicated by faster initial rise times for vessels in the islet core than for vessels on the perimeter (Figure 6A). However, a substantial number of islets (7 of 20; $35 \%$ ) showed no difference in rise times between the inner and outer vessels, indicating that flow was simultaneous to both areas (Figure 6B). One islet (5\%) demonstrated an "outer-to-inner" pattern, in which the rise times for the outer vessel regions came before those of the inner vessel regions (Figure 6C). These data, summarized in Figure 6D, indicate that in the majority of mouse islets, the central core of $\beta$ cells is upstream of non- $\beta$ cells, but in a substantial number of islets, the blood flow pattern is not dependent on islet cell type. 

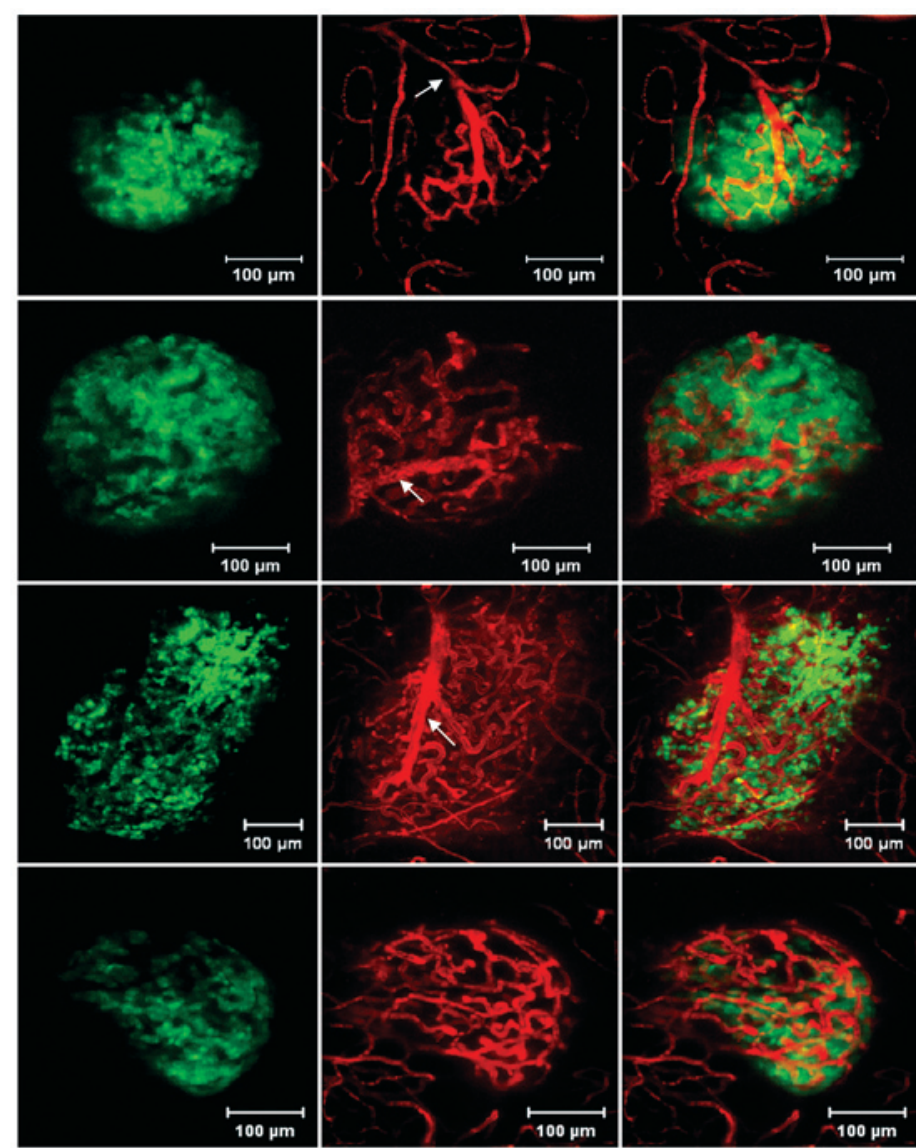

\section{Figure 3}

Through-focus projections of islets and their vasculature. Shown are GFP-labeled $\beta$ cells (left), the vasculature (middle), and the overlaid images (right) demonstrating the advantages of this visualization tool: making visible the full vascular tree in the islet, either in relation to the $\beta$ cells or alone. Larger vessels were found to have multiple smaller vessels extending from the vessel (arrows). Scale bars: $100 \mu \mathrm{m}$.

\section{Discussion}

We have established a method for in vivo dynamic imaging of pancreatic islet blood flow and have shown that it can be successfully used to resolve directional patterns of flow in the islet. We believe the novelty of our approach includes not only the in vivo experimental procedure, which incorporates the simultaneous labeling of the $\beta$ cells (MIP-GFP mice) and the vasculature (rhodamine dextran), but also the real-time 3D imaging capabilities that are essential for capturing the dynamics of blood flow. Prior studies that have investigated patterns of islet blood flow have led to contradictory results, perhaps because they provided only indirect measures of blood flow (16).

The 3 putative models of islet blood flow described in previous work have differing consequences regarding how blood flow is coupled to the distinct cellular arrangement of the islet and how it may impact intercellular communication. The 3 models can be described as (a) outer-to-inner, in which blood flows first to the non- $\beta$ cell perimeter and then to the $\beta$ cells in the islet core (13-16); (b) inner-to-outer, which proposes that the incoming arteries bypass the non $-\beta$ cell perimeter and first perfuse the $\beta$ cells $(8,12$, $18,19,28)$; and (c) top-to-bottom, in which the arteries perfuse blood from one side of the islet to the other regardless of cell type (21). The majority of evidence supporting these 3 models comes from vascular corrosion casting experiments, which are very high resolution but are performed on fixed samples. The few previous live-sample experiments have been complicated by a lack of $3 \mathrm{D}$ resolution, making it impossible to discern whether one vessel was in the same vertical plane as another (21).

Our results show 2 predominant flow patterns: inner-to-outer and top-to-bottom. The inner-to-outer flow pattern was the most prominent pattern, so it is likely that there are physiological consequences of differing cellular perfusion orders. This pattern supports the concept that insulin or other $\beta$ cell secretory products can have regulatory effects on downstream cells within the islet (i.e., $\alpha$ cells, which produce glucagon). Consequently, the order in which blood perfuses the islet may be pivotal in the regulation of blood glucose. Because only 1 islet demonstrated an outer-to-inner flow pattern, it is unlikely that secreted products of $\alpha$ cells influence $\beta$ cells.

The $35 \%$ of islets that showed a top-to-bottom flow pattern lends support to the model that proposes that islet blood flow is cell type independent. However, our results do not completely rule out the possibility that islet blood flow patterns are cell type dependent, because we do not know for certain the distribution of non- $\beta$ cell types within any given islet. It is also possible that irregular structures of islets in vivo, such as nonspherical islets and invaginations, could influence our definitions of what are inner and outer vessels in a way that could make some inner-to-outer 


\section{A}
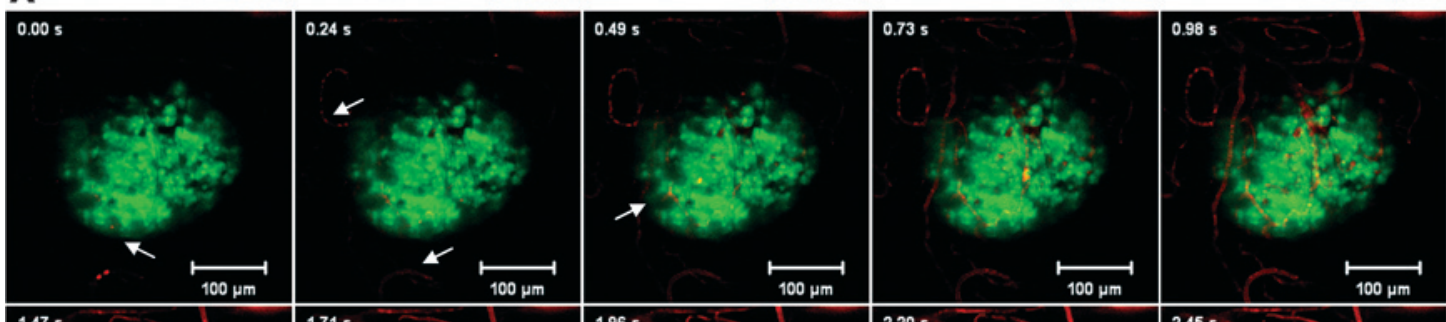

$1.22 \mathrm{~s}$
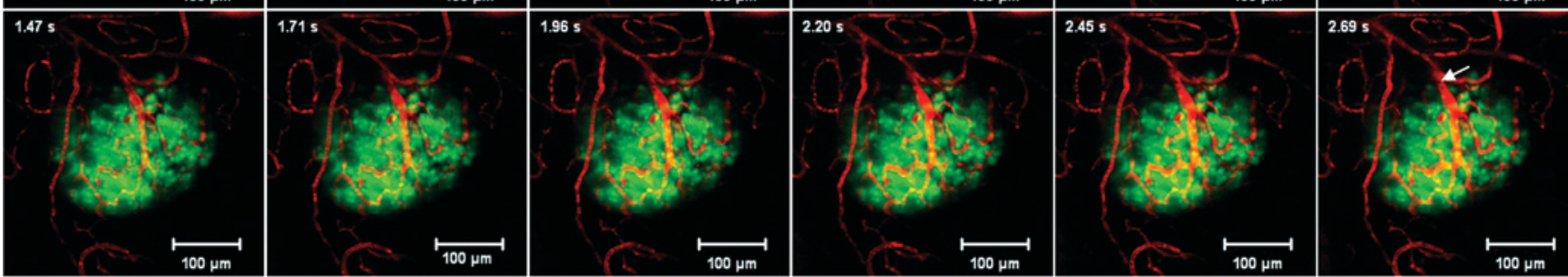

B
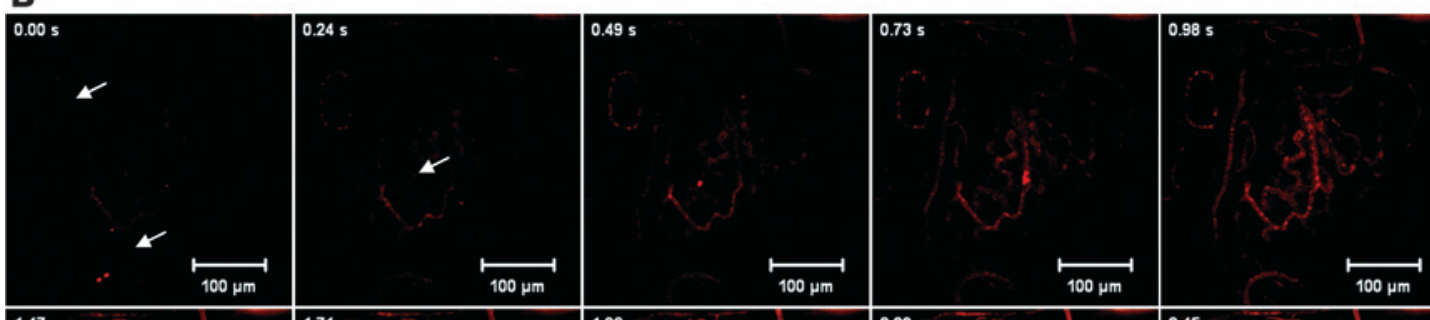

$1.22 \mathrm{~s}$
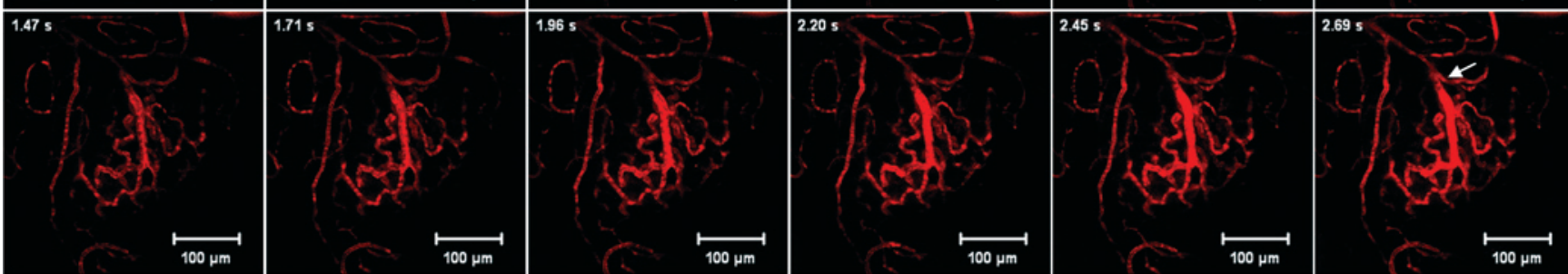

Figure 4

Real-time assessment of directional patterns of blood flow in the islet. Arrows denote vessel perfusion. (A) Time-resolved images (through-focus projection time series) showing the entry points of the rhodamine dextran (red) relative to the GFP-labeled $\beta$ cells (green) after a bolus injection of dextran. The time of acquisition is shown for each frame. Tracer flowed into several smaller vessels within the islet before it appeared in the large vessel, shown most clearly in the last panel. (B) Time-resolved images showing vessel perfusion order of the rhodamine dextran (red) only (without the $\beta$ cell mass), allowing for clear visualization of the sequential filling pattern of the vascular tree. These images further demonstrated that the largest vessel was not the first to be perfused and that blood entered first from 2 small arterioles. The last time point shows perfusion of this large vessel most clearly.

flow look more like top-to-bottom. However, it is unlikely that such an explanation would account for all $35 \%$ of the islets that exhibited this flow pattern. Finally, because of technical aspects involved in exteriorization of the intact pancreas, our findings are based on the examination of mainly the dorsal region of the pancreas and therefore only depict blood flow patterns of islets in this portion of the pancreas. The present study was also limited to those islets that reside near the pancreatic surface because of imaging depth limitations inherent to confocal microscopy. In the future it may be possible to increase this depth using endoscopic imaging or 2-photon excitation microscopy (29).

All prior islet blood flow models describe a large feeding artery that branches into capillaries. Although we did see this type of graduated branching, the larger vessels with numerous smaller vessels extending from them exclusively demonstrated efferent flow. We did find larger vessels with 1 or 2 branches connecting the islet showing afferent flow; however, the diameter of the vessel going into the islet was small and similar to that of islet capillaries. These data represent a comprehensive analysis that we believe was not possible to ascertain in prior studies because of limitations inherent in static in vitro studies.

The functional significance of these findings and why there are 2 predominant, distinct blood flow patterns will require further study. We did not note any recurring pattern of islet architecture, cellular composition, relationship to $\alpha$ islet cells or ducts, or location in a particular region of the pancreas to explain the different patterns of blood flow. The predominance of the inner-to-outer pattern suggests that intercellular communication may be important in the regulation of blood glucose, and therefore replicating the exact milieu of the islet as a cluster of different cell types may be significant for improving transplant studies. Although human islets have a cell type arrangement different from mouse islets (30), $\beta$ cells are thought to be grouped together with surrounding non- $\beta$ cells (6). Additional work is needed to integrate 
A

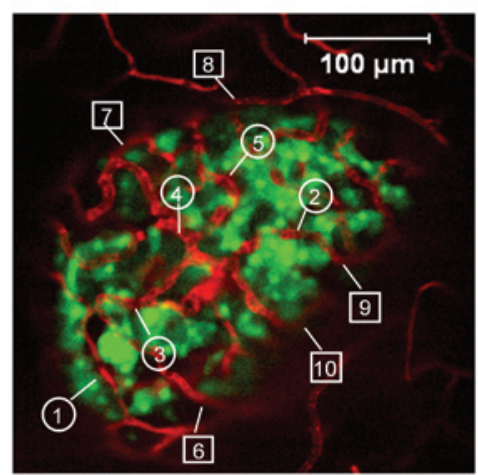

B

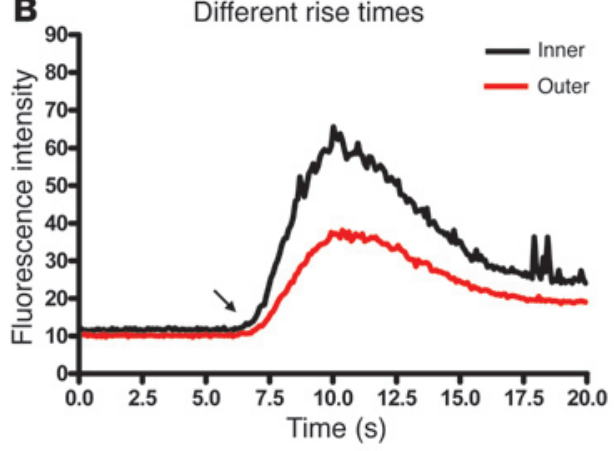

C

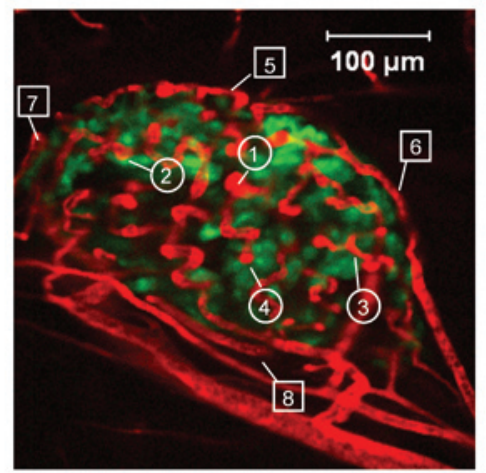

D

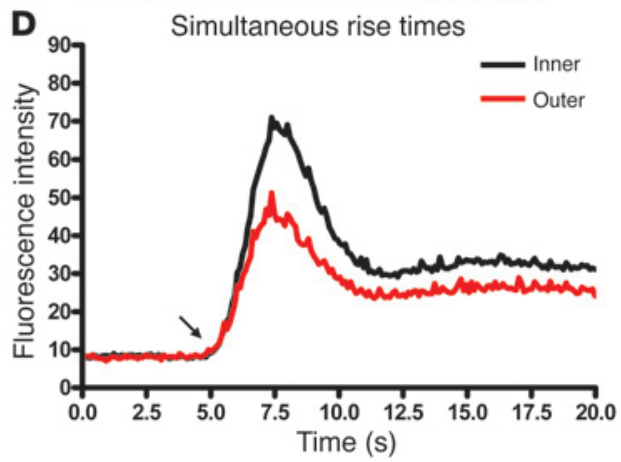

Figure 5

Temporal resolution analysis demonstrates more than 1 islet blood flow pattern. (A and $\mathbf{C}$ ) Two different islets (single-plane images), shown with inner (circles) and outer (squares) vessel regions selected and marked for fluorescence intensity measurement after a bolus of rhodamine dextran. Scale bars: $100 \mu \mathrm{m}$. (B and D) Average inner vessel fluorescent intensity time courses (throughout the $z$ plane) compared with average outer vessel fluorescence intensity time course for the islets in $\mathbf{A}$ and $\mathbf{C}$, respectively. Arrows denote rise times. Average rise time for the inner vessels was either (B) before that of outer vessels, as demonstrated by increased fluorescence intensity shown first by inner vessels, or (D) no different from outer vessels, which demonstrates that different islets had different perfusion patterns.

the current results with mouse islets to human islet blood flow. Understanding the factors that regulate islet blood flow may be important in defining the comprehensive mechanisms involved in islet hormone secretion.

This dynamic study of islet blood flow in time and space has provided information that was to our knowledge previously unattainable and demonstrates how critical in vivo imaging studies with sufficient temporal and spatial resolution are for understanding dynamic biological processes. In addition, data were examined directly and therefore not subject to potential artifacts arising from postprocessing or indirect analysis. Furthermore, these methods can be carried out using reagents and equipment that are now commercially available. The approach described in the present study should be useful for studying the in vivo regulation of pancreatic islet blood flow as well as the dynamics of blood flow in other organs.

\section{Methods}

Animals. Mice were housed in the animal care facility at Vanderbilt University and cared for according to the guidelines of the Vanderbilt Institutional Animal Care and Use Committee. The MIP-GFP transgenic mice were courtesy of M. Hara and G.I. Bell (University of Chicago, Chicago, Illinois, USA; ref. 24). We analyzed 103 islets in more than 90 mice for vascular structural patterns. Of those islets, 20 were used for the blood flow directionality studies. In most experiments, carotid artery and internal jugular vein catheters were placed prior to the study to allow for infusions during imaging; however, 5 of 20 mice were studied before catheterization was incorporated into the protocol. No significant difference was found in perfusion patterns between catheterized and noncatheterized mice (data not shown).

Pancreas exteriorization. Mice were anesthetized using intraperitoneal injection of a mixture of xylazine and ketamine $(20$ and $80 \mathrm{mg} / \mathrm{kg}$, respectively). An incision was made in the abdominal cavity so that the splenic end of the pancreas could be visualized. A coverslip wrapped with gauze bedding was placed gently on the abdominal cavity, and the pancreas-spleen connection was fixed over the bedding. The pancreas was kept moist with saline throughout the study. The mouse was then placed prone on the stage so that the pancreas was in contact with the coverslip. Mice were covered with a heating pad to maintain body temperature throughout the imaging period.

Imaging. The LSM 5 LIVE (Carl Zeiss Inc.) laser-scanning confocal microscope was used for imaging with a $\times 20,0.8$ NA planapochromat objective lens. The slit aperture was adjusted for each channel to allow for optimal signal-to-noise ratio and spatial resolution in each sample. After the pancreas was exposed on the microscope stage, islets were found by eye, using epifluorescence to identify the GFP-positive regions. Once the GFP-labeled $\beta$ cells were found, they were imaged using the 488 -nm diode laser line for excitation and a band-pass 495- to 525-nm emission filter for detection. Rhodamine dextran tracer $\left(2 \times 10^{6} \mathrm{MW}\right.$; Invitrogen $)$ was then injected into the vasculature via the ocular plexus in order to visualize blood flow, and this was imaged using the 532-nm diode laser line for 
A

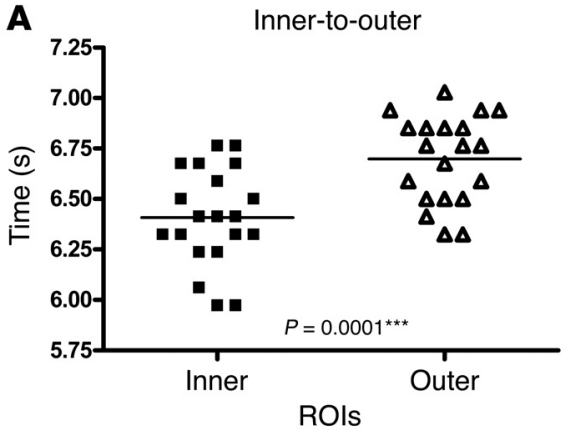

C

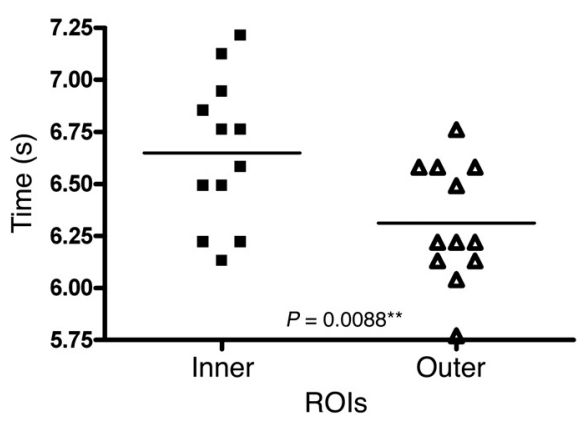

B

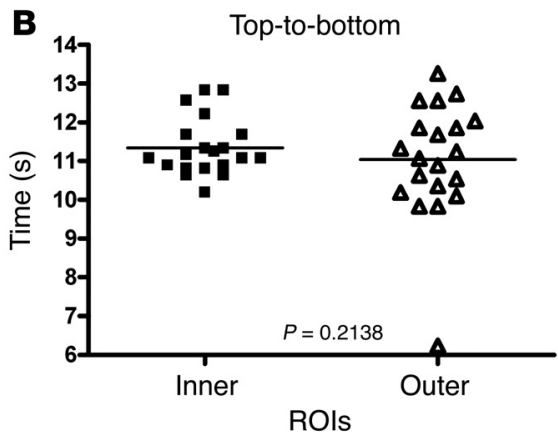

D

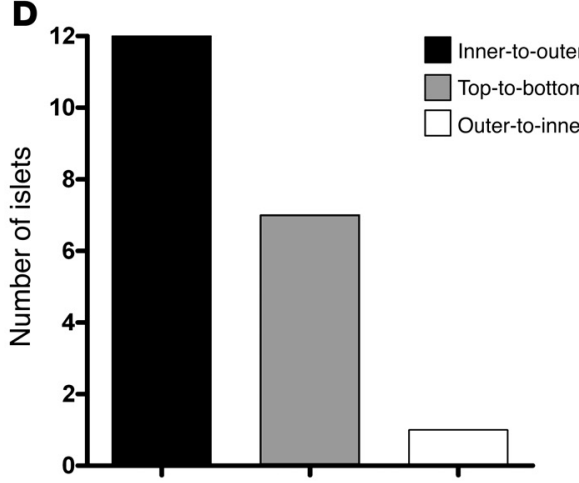

Figure 6

Quantification of islet perfusion patterns. Vertical scatter plots show quantification of the initial fluorescence intensity rise time for inner (squares) and outer (triangles) vessels after rhodamine dextran bolus injection. (A) Representative islet in which mean rise time for inner vessels was prior to that for outer vessels (inner-to-outer). (B) Representative islet in which mean rise time was similar for inner and outer vessels (top-tobottom). (C) Representative islet in which mean rise time for inner vessels was after that for outer vessels (outer-to-inner). (D) Number of islets demonstrating each perfusion pattern.

excitation and a band-pass 560- to 675-nm emission filter for detection. To assess islet blood flow directionality, $z$ stacks were made in increments of $4 \mu \mathrm{m}$ over an average of $25-40 \mu \mathrm{m}$ in depth. Images were acquired at a rate of more than $70 \mathrm{fps}$, e.g., 10 images at different focal planes could be repeated 7 times per second. The microscope focal plane nearest to the bottom of the islet (the side closest to the coverglass) was referred to as the top boundary, and the bottom boundary was selected from the focal plane in the image furthest from the coverglass.

Ocular plexus injection. After placement of the mouse on the microscope stage, an islet was identified close to the surface for the best resolution possible. The imaging parameters were established, imaging commenced at more than $70 \mathrm{fps}$, and $50 \mu \mathrm{l}$ rhodamine dextran was injected into the ocular plexus. After the filling of the vasculature, the imaging was saved and analyzed.

Image analysis. All images were analyzed using both linear unmixing, a numerical technique for efficient spectral separation of the 2 fluorescence channels, and ROI analysis software (Zeiss LSM5, version 4.0; Carl Zeiss Inc.). Figures have been contrast enhanced to aid visualization; however, all data analysis was conducted on the original raw images before any contrast enhancement.

Fluorescence intensity rise times. We determined the arrival time of a bolus at each of the selected ROIs by finding the first time point that met both of the following criteria: (a) the ROI had an intensity value greater than the arithmetic mean; and (b) intensities at each following time point increased monotonically until the first local maxima was reached. We used the intensity mean as a trigger value to minimize the effects of background noise. The time dimension of the dynamic fluorescence intensity graphs was normalized so that the starting point of increase was on a similar timeline. Time before injection varied as a result of technical aspects of injection. Comparison included inner versus outer points per islet; therefore, normalizing the 0 time point did not affect or bias results.

Statistics. To analyze comparison of inner and outer points, 1-tailed Student's $t$ tests were used. $P$ values less than 0.05 were considered significant.

\section{Acknowledgments}

We thank Todd Salter for programming assistance as well as Tasneem Ansari and Carlo Malabanan from the Vanderbilt Mouse Metabolic Phenotyping Center. This study was supported by NIH grants DK69603, DK68764, DK53434, RR22620, DK66636, DK63439, DK07563, and DK07563; by a Merit Review Award from the VA Research Service; by a grant from the Juvenile Diabetes Research Foundation International; by the Vanderbilt Mouse Metabolic Phenotyping Center (NIH grant DK59637); by a mentored fellowship award from the American Diabetes Association; and by the Vanderbilt Diabetes Research and Training Center (NIH grant DK20593). Imaging and data analysis were performed in part through the use of the Vanderbilt University Medical Center Cell Imaging Shared Resource, supported by NIH grants CA68485, DK20593, DK58404, HD15052, DK59637, and EY08126.

Received for publication May 15, 2008, and accepted in revised form September 3, 2008. 
Address correspondence to: Alvin C. Powers, Department of Medicine, Division of Diabetes, Endocrinology, and Metabolism, Room 7465, Medical Research Building IV, Vanderbilt University, Nashville, Tennessee 37232, USA. Phone: (615) 322-7004; Fax: (615) 343-0172;
E-mail: al.powers@vanderbilt.edu. Or to: David W. Piston, Department of Molecular Physiology and Biophysics, 702 Light Hall, Vanderbilt University, Nashville, Tennessee 37232, USA. Phone: (615) 322-7030; Fax: (615) 322-7236; E-mail: dave.piston@vanderbilt.edu.
1. Li, X., et al. 2006. Islet microvasculature in islet hyperplasia and failure in a model of type 2 diabetes. Diabetes. 55:2965-2973.

2. Anneren, C., Welsh, M., and Jansson, L. 2007. Glucose intolerance and reduced islet blood flow in transgenic mice expressing the FRK tyrosine kinase under the control of the rat insulin promoter. Am. J. Physiol. Endocrinol. Metab. 292:E1183-E1190.

3. Svensson, A.M., Ostenson, C.G., and Jansson, L. 2000. Age-induced changes in pancreatic islet blood flow: evidence for an impaired regulation in diabetic GK rats. Am. J. Physiol. Endocrinol. Metab. 279:E1139-E1144.

4. Jansson, L., and Carlsson, P.O. 2002. Graft vascular function after transplantation of pancreatic islets. Diabetologia. 45:749-763.

5. Brissova, M., et al. 2006. Pancreatic islet production of vascular endothelial growth factor-a is essential for islet vascularization, revascularization, and function. Diabetes. 55:2974-2985.

6. Weir, G.C., and Bonner-Weir, S. 1990. Islets of Langerhans: the puzzle of intraislet interactions and their relevance to diabetes. J. Clin. Invest. 85:983-987.

7. Orci, L., and Unger, R.H. 1975. Functional subdivision of islets of Langerhans and possible role of D cells. Lancet. 2:1243-1244.

8. Bonner-Weir, S. 1993. The microvasculature of the pancreas, with emphasis on that of the islets of langerhans. In The pancreas: biology, pathobiology, and disease. Vay Liang W. Go, editor. Raven Press. New York, New York, USA. 759-768.

9. Xu, E., et al. 2006. Intra-islet insulin suppresses glucagon release via GABA-GABAA receptor system. Cell Metab. 3:47-58.

10. Ravier, M.A., and Rutter, G.A. 2005. Glucose or insulin, but not zinc ions, inhibit glucagon secretion from mouse pancreatic alpha-cells. Diabetes.
54:1789-1797.

11. Stagner, J.I., Samols, E., and Marks, V. 1989. The anterograde and retrograde infusion of glucagon antibodies suggests that A cells are vascularly perfused before D cells within the rat islet. Diabetologia. 32:203-206.

12. Stagner, J.I., and Samols, E. 1986. Retrograde perfusion as a model for testing the relative effects of glucose versus insulin on the A cell. J. Clin. Invest. 77:1034-1037.

13. Murakami, T., et al. 1993. The insulo-acinar portal and insulo-venous drainage systems in the pancreas of the mouse, dog, monkey and certain other animals: a scanning electron microscopic study of corrosion casts. Arch. Histol. Cytol. 56:127-147.

14. Miyake, T., Murakami, T., and Ohtsuka, A. 1992. Incomplete vascular casting for a scanning electron microscope study of the microcirculatory patterns in the rat pancreas. Arch. Histol. Cytol. 55:397-406.

15. Ohtani, O. 1984. Review of scanning electron and light microscopic methods in microcirculation research and their application in pancreatic studies. Scan. Electron Microsc. (Pt. 2):653-661.

16. Brunicardi, F.C., et al. 1996. Microcirculation of the islets of Langerhans. Long Beach Veterans Administration Regional Medical Education Center Symposium. Diabetes. 45:385-392.

17. Ballian, N., and Brunicardi, F.C. 2007. Islet vasculature as a regulator of endocrine pancreas function. World J. Surg. 31:705-714.

18. Bonner-Weir, S., and Orci, L. 1982. New perspectives on the microvasculature of the islets of Langerhans in the rat. Diabetes. 31:883-889.

19. Samols, E., Stagner, J.I., Ewart, R.B., and Marks, V. 1988. The order of islet microvascular cellular perfusion is B----A----D in the perfused rat pancreas. J. Clin. Invest. 82:350-353.

20. Aharinejad, S., et al. 1993. Scanning and trans- mission electron microscopy and high resolution intravital video-microscopy of capillaries in the mouse exocrine pancreas, with special emphasis on endothelial cells. Anat. Rec. 237:163-177.

21. Liu, Y.M., Guth, P.H., Kaneko, K., Livingston, E.H., and Brunicardi, F.C. 1993. Dynamic in vivo observation of rat islet microcirculation. Pancreas. 8:15-21.

22. Cahalan, M.D., Parker, I., Wei, S.H., and Miller, M.J. 2002. Two-photon tissue imaging: seeing the immune system in a fresh light. Nat. Rev. Immunol. 2:872-880.

23. Speier, S., et al. 2008. Noninvasive in vivo imaging of pancreatic islet cell biology. Nat. Med. 14:574-578.

24. Hara, M., et al. 2003. Transgenic mice with green fluorescent protein-labeled pancreatic beta-cells. Am. J. Physiol. Endocrinol Metab. 284:E177-E183.

25. Dickinson, M.E. 2006. Multimodal imaging of mouse development: tools for the postgenomic era. Dev. Dyn. 235:2386-2400.

26. Megason, S.G., and Fraser, S.E. 2007. Imaging in systems biology. Cell. 130:784-795.

27. Kleinfeld, D., Mitra, P.P., Helmchen, F., and Denk, W. 1998. Fluctuations and stimulus-induced changes in blood flow observed in individual capillaries in layers 2 through 4 of rat neocortex. Proc. Natl. Acad. Sci. U. S. A. 95:15741-15746.

28. Samols, E., and Stagner, J.I. 1990. Islet somatostatin-microvascular, paracrine, and pulsatile regulation. Metabolism. 39:55-60.

29. Benninger, R.K., Hao, M., Piston, D.W. 2008. Multiphoton excitation imaging of dynamic processes in living cells and tissues. Rev. Physiol. Biochem. Pharmacol. 160:71-92.

30. Brissova, M., et al. 2005. Assessment of human pancreatic islet architecture and composition by laser scanning confocal microscopy. J. Histochem. Cytochem. 53:1087-1097. 